\title{
Robust Digital Control of Flexible Transmissions Using the Combined Pole Placement/Sensitivity Function Shaping Method*
}

\author{
I. D. Landau, A. Karimi, A. Voda and D. Rey
}

Laboratoire d'Automatique de Grenoble (INPG/CNRS) and Groupement de Recherche Automatique (CNRS), Ecole Nationale Supérieure d'Ingénieurs Electriciens de Grenoble Domaine Universitaire, Grenoble, France

The control of very flexible transmissions in the presence of load variations is a challenging control problem. In this paper it is shown that based on identified discrete time models a robust digital linear controller can be designed using the combined pole placement method with sensitivity function shaping in the frequency domain. This controller provides satisfactory performance for a large range of load variations.

Keywords: Digital control; Robust control; Pole placement; Loop shaping; Flexible systems

\section{Introduction}

A robust linear digital controller is designed to solve the benchmark problem presented in [1]. This controller provides satisfactory performance for a large range of load variations. The controller design methodology is based on pole placement combined with the sensitivity function shaping.

The computation of the controller requires the specification of the desired closed loop poles (the nominal stability problem) and of some fixed parts of the controller for the rejection of disturbances at various frequencies (the nominal performance problem). The robustness of the closed loop system with respect to the plant model uncertainties (the robust stability and

\footnotetext{
*Work supported by G.R. Automatique (CNRS).

Paper presented at ECC 95, Rome, 5-8 September 1995.
}

Correspondence and offprint requests to: I. D. Landau, Laboratoire d'Automatique de Grenoble, Domaine Universitaire, 38402 SaintMartin d'Hères, France. E-mail: landau@lag.grenet.fr robust performance problem) will depend on these specifications. A methodology is therefore needed for correctly specifying the desired closed loop poles and the fixed parts of the controller in order to assure the 'nominal performance', and the 'robust stability' of the closed loop for given classes of plant model uncertainties.

The sensitivity functions, particularly the output sensitivity function, are key indicators for the nominal and robust performance as well as for the robust stability of the closed loop system.

The inverse of the maximum value of the output sensitivity function, i.e. the inverse of its $H_{\infty}$ norm, gives the minimum distance between the Nyquist plot of the open loop system and the critical point $[-1, j 0]$. This quantity called the modulus margin, is a much more significant robustness indicator than the phase and gain margins. On the other hand, conditions for assuring a certain 'delay' margin which is also a very important robustness indicator (particularly in the high frequency region) can also be expressed in terms of the shape of the output sensitivity function. It seems therefore reasonable to combine the pole placement with the shaping of the output sensitivity function (or eventually with other sensitivity functions) in order to design robust digital controllers for SISO plants. This method has been applied to a large number of real systems $[2,3]$.

The paper is organised as follows: Section 2 reviews the pole placement technique. Section 3 gives the various sensitivity functions associated with the pole placement design. In Section 4 a template for the output sensitivity function is defined. The shaping

Received 15 May 1995; Accepted in revised form 28 July 1995 Recommended by S. Graebe and D. Clarke 
method for the sensitivity function is described in Section 5. The controller design is presented in Section 6 and the simulation results are given in Section 7 .

\section{Pole Placement [4]}

The discrete time plant is described by the following transfer operator:

$$
H\left(q^{-1}\right)=\frac{q^{-d} B\left(q^{-1}\right)}{A\left(q^{-1}\right)}
$$

where $q^{-1}$ is the backward shift operator $\left(y(t-1)=q^{-1} y(t)\right), d$ is the integer number of sampling periods $\left(T_{\mathrm{S}}\right)$ contained in the plant pure time delay and:

$$
\begin{aligned}
& A\left(q^{-1}\right)=1+a_{1} q^{-1}+\cdots a_{n_{A}} q^{-n_{A}} \\
& B\left(q^{-1}\right)=b_{1} q^{-1}+b_{2} q^{-2}+\cdots b_{n_{B}} q^{-n_{B}}
\end{aligned}
$$

It is assumed that the polynomials $A\left(q^{-1}\right)$ and $B\left(q^{-1}\right)$ do not have common factors.

The canonical form of the RST controller is given by:

$$
S\left(q^{-1}\right) u(t)=T\left(q^{-1}\right) y^{*}(t+d+1)-R\left(q^{-1}\right) y(t)
$$

where $u(t)$ is the plant input, $y(t)$ is the plant output and $y^{*}(t+d+1)$ is the desired tracking (reference) trajectory. This trajectory may be generated by a tracking reference model.

$$
y^{*}(t+d+1)=\frac{B_{\mathrm{m}}\left(q^{-1}\right)}{A_{\mathrm{m}}\left(q^{-1}\right)} r(t)
$$

where $r(t)$ is the reference signal.

The closed loop transfer function between the reference trajectory and the plant output is given by:

$$
H_{\mathrm{CL}}\left(z^{-1}\right)=\frac{z^{-d} B\left(z^{-1}\right) T\left(z^{-1}\right)}{P\left(z^{-1}\right)}
$$

where

$$
\begin{aligned}
P\left(z^{-1}\right) & =A\left(z^{-1}\right) S\left(z^{-1}\right)+z^{-d} B\left(z^{-1}\right) R\left(z^{-1}\right) \\
& =P_{D}\left(z^{-1}\right) \cdot P_{F}\left(z^{-1}\right)
\end{aligned}
$$

defines the closed loop poles.

In Eq. (1) $P_{D}\left(z^{-1}\right)$ corresponds to the dominant closed loop poles chosen to satisfy the desired regulation performance; $P_{F}\left(z^{-1}\right)$ corresponds to the auxiliary poles which can be introduced either for filtering effects in certain frequency regions or for reducing the stress on the actuator as well as for improving the robustness of the closed loop system (as will be shown subsequently). For different reasons (i.e. disturbance rejection, signal blocking) the polynomials
$R\left(z^{-1}\right)$ and $S\left(z^{-1}\right)$ generally contain some fixed parts which are specified before solving Eq. (1) (for example the polynomial $S\left(z^{-1}\right)$ should contain a term $\left(1-z^{-1}\right)$ for a null steady state error).

In order to take into account these pre-specified parts, the polynomials $R\left(z^{-1}\right)$ and $S\left(z^{-1}\right)$ are factored as:

$$
\begin{aligned}
& R\left(z^{-1}\right)=R^{\prime}\left(z^{-1}\right) H_{\mathrm{R}}\left(z^{-1}\right) \\
& S\left(z^{-1}\right)=S^{\prime}\left(z^{-1}\right) H_{\mathrm{S}}\left(z^{-1}\right)
\end{aligned}
$$

where $H_{\mathrm{R}}\left(z^{-1}\right)$ and $H_{\mathrm{S}}\left(z^{-1}\right)$ are the pre-specified polynomials. The closed loop poles are given in this case by:

$$
\begin{aligned}
P\left(z^{-1}\right)= & A\left(z^{-1}\right) H_{\mathrm{S}}\left(z^{-1}\right) S^{\prime}\left(z^{-1}\right) \\
& +z^{-d} B\left(z^{-1}\right) H_{\mathrm{R}}\left(z^{-1}\right) R^{\prime}\left(z^{-1}\right)
\end{aligned}
$$

The design procedure can be summarised as follows:

1. Choose the desired closed loop poles $P\left(z^{-1}\right)$, the fixed parts of the controller $H_{\mathrm{R}}\left(z^{-1}\right), H_{\mathrm{S}}\left(z^{-1}\right)$ and the desired tracking dynamics $B_{\mathrm{m}}\left(z^{0-1}\right) / A_{\mathrm{m}}\left(z^{-1}\right)$.

2. Compute $S^{\prime}\left(z^{-1}\right)$ and $R^{\prime}\left(z^{-1}\right)$ by solving Eq. (4) and consequently compute $S\left(z^{-1}\right)$ and $R\left(z^{-1}\right)$ using Eqs (2) and (3).

3. Compute the pre-filter $T\left(z^{-1}\right)=G P\left(z^{-1}\right)$

where

$$
G=\left\{\frac{1 / B(1) \text { if } B(1) \neq 0}{1 \text { if } B(1)=0}\right\}
$$

This choice leads to the following transfer function between the reference and the plant output:

$$
H_{\mathrm{yr}}\left(z^{-1}\right)=\frac{z^{-d} B_{\mathrm{m}}\left(z^{-1}\right)}{A_{\mathrm{m}}\left(z^{-1}\right)} \cdot \frac{B\left(z^{-1}\right)}{B(1)}
$$

Another method consists of taking $T\left(z^{-1}\right)=R(1)$

$$
H_{\mathrm{yr}}\left(z^{-1}\right)=\frac{z^{-d} B_{\mathrm{m}}\left(z^{-1}\right)}{A_{\mathrm{m}}\left(z^{-1}\right)} \cdot \frac{B\left(z^{-1}\right)}{P\left(z^{-1}\right)} \cdot \frac{P(1)}{B(1)}
$$

\section{The Sensitivity Functions}

The sensitivity functions play a crucial role in the robustness analysis of the closed loop system with respect to modelling errors. These functions will be 'shaped' in order to assure 'nominal performance' for the rejection of the disturbances and the stability of the closed loop system in the presence of model mismatch. Two types of disturbances are considered: the output disturbance and the measurement noise. The 


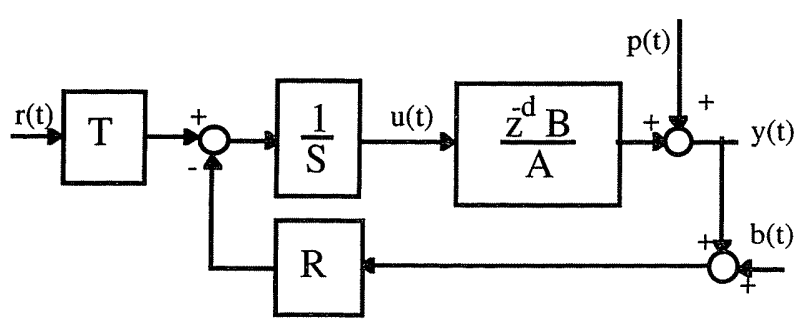

Fig. 1. Closed loop system with a RST controller in presence of output disturbances and measurement noise.

various sensitivity functions can be established from Fig. 1.

The transfer functions between the disturbance $p(t)$ and the plant output $y(t)$ (output sensitivity function), between $p(t)$ and the plant input $u(t)$ (input sensitivity function) and between the measurement noise $b(t)$ and $y(t)$ (noise sensitivity function) are given respectively by:

$$
\begin{aligned}
S_{\mathrm{yp}}\left(z^{-1}\right) & =\frac{A\left(z^{-1}\right) S\left(z^{-1}\right)}{A\left(z^{-1}\right) S\left(z^{-1}\right)+z^{-d} B\left(z^{-1}\right) R\left(z^{-1}\right)} \\
& =\frac{A\left(z^{-1}\right) S\left(z^{-1}\right)}{P\left(z^{-1}\right)} \\
S_{\mathrm{up}}\left(z^{-1}\right) & =\frac{-A\left(z^{-1}\right) R\left(z^{-1}\right)}{A\left(z^{-1}\right) S\left(z^{-1}\right)+z^{-d} B\left(z^{-1}\right) R\left(z^{-1}\right)} \\
& =\frac{-A\left(z^{-1}\right) R\left(z^{-1}\right)}{P\left(z^{-1}\right)} \\
S_{\mathrm{yb}}\left(z^{-1}\right) & =\frac{-z^{-d} B\left(z^{-1}\right) R\left(z^{-1}\right)}{A\left(z^{-1}\right) S\left(z^{-1}\right)+z^{-d} B\left(z^{-1}\right) R\left(z^{-1}\right)} \\
& =\frac{-z^{-d} B\left(z^{-1}\right) R\left(z^{-1}\right)}{P\left(z^{-1}\right)}
\end{aligned}
$$

\section{Definition of a 'Template' for the Output Sensitivity Function}

Using the small gain theorem and various representations of the plant uncertainties, the modulus margin and the delay margin can be converted into 'robust stability' conditions. On the other hand the robust stability conditions allow the definition of 'upper' templates for the modulus of the various sensitivity functions $[2,5,6]$.

For a 'delay margin' of one sampling period the robust stability condition is expressed as follows [7]:

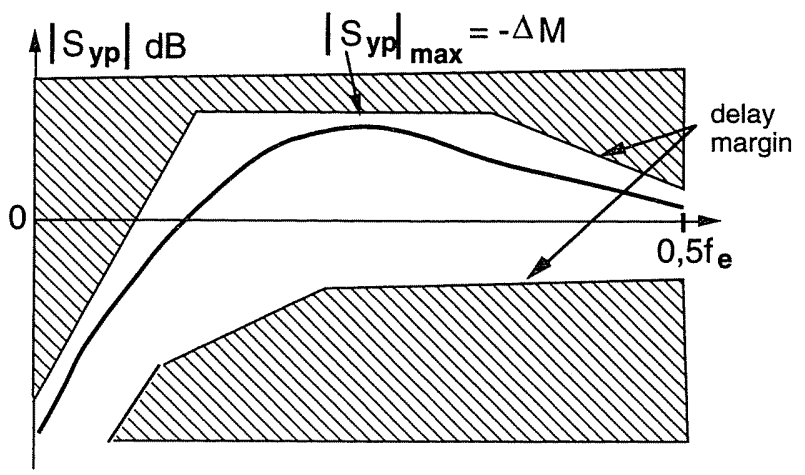

Fig. 2. Desired template for the output sensitivity function (the case of low frequencies disturbance rejection).

$$
\begin{aligned}
& \left|S_{\mathrm{yb}}\left(z^{-1}\right)\right|<\left|\left(1-z^{-1}\right)\right|^{-1} \\
& z=e^{-j \omega}, 0 \leq \omega \leq \pi
\end{aligned}
$$

But, we have:

$$
S_{\mathrm{yp}}\left(z^{-1}\right)-S_{\mathrm{yb}}\left(z^{-1}\right)=1
$$

and therefore

$$
1-\left|S_{\mathrm{yb}}\left(z^{-1}\right)\right|<\left|S_{\mathrm{yp}}\left(z^{-1}\right)\right|<1+\left|S_{\mathrm{yb}}\left(z^{-1}\right)\right|
$$

If $S_{\mathrm{yb}}\left(z^{-1}\right)$ satisfies condition (8) then $S_{\mathrm{yp}}\left(z^{-1}\right)$ will satisfy the following condition :

$$
\begin{aligned}
& 1-\left|1-z^{-1}\right|^{-1}<S_{\text {yp }}\left(z^{-1}\right)|<1+| 1-\left.\dot{z}^{-1}\right|^{-1} ; \\
& z=e^{-j \omega}, 0 \leq \omega \leq \pi
\end{aligned}
$$

Therefore in order to assure the delay margin $\Delta \tau=T_{\mathrm{s}}$, it is required that the modulus of $S_{\mathrm{yp}}\left(z^{-1}\right)$ lies inside a 'tube' defined by a lower template $\left|W^{-1}\right|_{\text {inf }}=1-\left|1-z^{-1}\right|^{-1}$ and an upper template defined by $\left|W^{-1}\right|_{\text {sup }}=1+\left|1-z^{-1}\right|^{-1}$.

The 'nominal performance' requirements and the 'robust stability' conditions lead to the definition of a desired 'template' for the sensitivity functions. We will subsequently consider the definition of such a template for the output sensitivity function. The desired template takes in general the form shown in Fig. 2.

Regarding the robust stability, the chosen modulus margin will define the maximum value of the modulus of the output sensitivity function (upper template) and the chosen delay margin will define an upper and a lower template starting for example around $0.15 f_{\mathrm{s}}\left(\right.$ for $\Delta \tau=T_{\mathrm{s}}$ ).

\section{Shaping the Sensitivity Function}

The dominant poles $P_{\mathrm{D}}\left(z^{-1}\right)$, the auxiliary poles $P_{\mathrm{F}}\left(z^{-1}\right)$ and the two filters $H_{\mathrm{R}}\left(z^{-1}\right)$ and $H_{\mathrm{S}}\left(z^{-1}\right)$ 
are the tools for shaping the various sensitivity functions in order to meet the specifications defined by the desired templates. Placing a pair of complex zeros in $H_{\mathrm{S}}\left(z^{-1}\right)$ decreases the magnitude of the modulus of the sensitivity function around the frequencies of these zeros. Introducing a pair of pure imaginary zeros at a certain frequency in $H_{\mathrm{R}}\left(z^{-1}\right)$ will make $\left|S_{\mathrm{yp}}\right|=1$ and $\left|S_{\mathrm{up}}\right|=0$ at this frequency. Complex zeros in $H_{\mathrm{R}}\left(z^{-1}\right)$ will bring $\left|S_{\mathrm{yp}}\right|$ closer to the $0 \mathrm{~dB}$ axis and will reduce $\left|S_{\text {up }}\right|$. Putting some asymptotically stable real high frequency poles in $P\left(z^{-1}\right)$ will cause an attenuation of $\left|S_{\mathrm{yp}}\right|$ in the domain of attenuation of $1 / P_{\mathrm{F}}\left(z^{-1}\right)$. Placing a pair of complex poles at a certain frequency in $P_{\mathrm{F}}\left(z^{-1}\right)$ will lead to an increase in $\left|S_{\mathrm{yp}}\right|$ around this frequency.

The underlying philosophy of the design is to choose the closed loop poles and the fixed parts of the controller in order to bring the sensitivity function within the defined template. The shaping method which is an iterative procedure (generally converging in few steps) is as follows:

\section{Step I}

- Choose the dominant closed loop poles of $P\left(z^{-1}\right)$ and the fixed part of $R\left(z^{-1}\right)$ and $S\left(z^{-1}\right)$ in order to meet the nominal performance specifications.

- Compute the controller.

- Check the shape of the output sensitivity function. If the upper bound of the template is not satisfied, generally two situations are identified:

(a) The maximum of the sensitivity function is located in the frequency range next to the attenuation band. In this case, go to step II;

(b) The maximum of the sensitivity function is located in the high frequency range (near 0.5 $f_{\mathrm{s}}$ ). In this case jump to step III.

\section{Step II}

- Add a pair of complex zeros to $H_{\mathrm{S}}\left(z^{-1}\right)$ :

$$
H_{\mathrm{S}}\left(z^{-1}\right)=1+\alpha_{1} z^{-1}+\alpha_{2} z^{-2}
$$

The frequency of these zeros is chosen close to the frequency where the maximum of the $\left|S_{\mathrm{yp}}\right|$ occurs (between the maximum frequency of the attenuation band and the frequency where $\left|S_{\mathrm{yp}}\right|_{\max }$ occurs). The damping factor is chosen such that the introduced attenuation brings $\left|S_{\mathrm{yp}}\right|_{\max }$ below the admissible value. Typical values are between $\zeta=0.3-0.8$.

- Recompute the controller. If $\left|S_{\mathrm{yp}}\right|$ is too large in the high frequency range, go to step III.
Remark: The effect of these additional zeros are in general:

(a) A reduction in the modulus of the sensitivity function in the region next to the attenuation band.

(b) An increase in the maximum of the modulus of the sensitivity function in the high frequency range (in general its maximum is shifted in the high frequency range).

(c) An increase in the attenuation band.

\section{Step III}

- Add auxiliary high frequency poles of the form:

$$
P_{\mathrm{F}}\left(z^{-1}\right)=\left(1-p_{1} z^{-1}\right)^{n_{\mathrm{F}}} ; 0.05 \leq p_{1} \leq 0.5
$$

where

$$
\begin{aligned}
& n_{\mathrm{F}} \leq n_{\mathrm{P}}-n_{\mathrm{D}} ; n_{\mathrm{P}}=(\operatorname{deg} P)_{\max } \\
& n_{\mathrm{D}}=\operatorname{deg} P_{\mathrm{D}}
\end{aligned}
$$

with increasing values of $p_{1}$ starting from 0.05 till the specifications on the $\left|S_{\mathrm{yp}}\right|$ in the high frequency range are met. It should be mentioned that high frequency auxiliary poles shift, in general, the maximum of the $\left|S_{\mathrm{yp}}\right|$ towards the lower frequency range. If the $\left|S_{\mathrm{yp}}\right|_{\max }$ is satisfactory stop here, if not go to step IV.

\section{Step IV}

This step is similar to step II:

- If the new maximum is close to the one resulting after step I, change accordingly the frequency and the damping of the zeros introduced in $H_{\mathrm{S}}$.

- If the new maximum is at a significantly different frequency, add a new pair of complex zeros in $H_{\mathrm{S}}\left(z^{-1}\right)$ at a frequency close to the maximum of $\left|S_{\mathrm{yp}}\right|$. The damping is chosen in order to bring $\left|S_{\text {yp }}\right|_{\text {max }}$ below the maximum acceptable value. If the results are unsatisfactory, go to step V.

\section{Step V}

- The value of the real auxiliary poles (defined at step III) can be increased.

- If necessary, the dominant poles also may be slowed down.

*End of the procedure for the shaping of the output sensitivity function.

*Check the input sensitivity function. Add if necessary high frequency complex zeros in $H_{\mathrm{R}}\left(z^{-1}\right)$ (often at $0.5 f_{\mathrm{s}}$ ). 


\section{Controller Design for the Benchmark Problem}

The analysis of the frequency characteristics of the models under different loads shows that [1]:

1. The unloaded model is the most difficult to control because it has the lowest damping factors.

2. Very large gain variations (around $20 \mathrm{~dB}$ ) take place between the frequencies corresponding to the first vibration mode of the full load model and that of the unloaded model.

3. If the synthesis of the controller is carried out for the unloaded case, it is observed that at frequencies above the first vibration mode of the no load model, the process gain will be lower in the presence of load. This indicates that this region will not raise problems for the stability of the closed loop system.

From this analysis, it is found that the critical frequency zone both for robust stability and robust performance is located at frequencies below the first vibration mode of the unloaded model $\left(\omega<12.6 \mathrm{rad} / \mathrm{s} ; f / f_{\mathrm{s}}<0.1\right)$.

In the first step, a controller with integrator, based on the pole placement technique is computed for the no load model. The dominant poles correspond to a second order system having a natural frequency equal to that of the first vibration mode of the full load model with a damping factor of 0.9 .

The frequency characteristics of the sensitivity function are given in Fig. 3 (curve A). It is observed that the output sensitivity function is almost placed in the defined template therefore the controller meets the conditions for the nominal model. The lower template is computed for a delay margin of one sampling period whereas the desired delay margin is $0.8 T_{\mathrm{s}}$. Therefore the small violation of the lower template is not very important since the required delay margin is assured.

For robust stability, the model uncertainty should be analysed. If the unloaded model is chosen as the nominal plant the additive uncertainty will be given by:

$$
\begin{aligned}
& \left|W_{a}(j \omega)\right|=\max \left\{\left|P_{\mathrm{U}}(j \omega)-P_{\mathrm{H}}(j \omega)\right|,\right. \\
& \left.\left|P_{\mathrm{U}}(j \omega)-P_{\mathrm{F}}(j \omega)\right|\right\}
\end{aligned}
$$

where $P_{\mathrm{U}}, P_{\mathrm{H}}$ and $P_{\mathrm{F}}$ are the no load, half load and full load models, respectively. Using the small gain theorem, the sufficient condition for robust stability for all of the plant will be as follows [2]:

$$
\left|S_{\mathrm{up}}(j \omega)\right|<\left|W_{a}^{-1}(j \omega)\right|
$$

Figure 4 shows the magnitude of $S_{\mathrm{up}}(j \omega)$ and $W_{a}^{-1}(j \omega)$ respectively as curves $\mathrm{A}$ and $\mathrm{W}$. To satisfy Eq. (9) a pair of zeros with a frequency near the minimum value of $W_{a}^{-1}(j \omega)$ should be added in either $H_{\mathrm{R}}$ or $H_{\mathrm{S}}$ in order to decrease the magnitude of $S_{\mathrm{up}}$. Adding this pair of zeros in $H_{\mathrm{S}}$ causes a peak value in $S_{\mathrm{yp}}$ at high frequency. To solve this problem the algorithm presented in Section 5 can be used. Figure 4 (curve C) shows $S_{\text {up }}$ after introducing a pair of complex zeros in $H_{\mathrm{S}}\left(\omega_{0}=5.8 \mathrm{rad} / \mathrm{s}\right.$ and $\left.\zeta=0.4\right)$ and shaping $S_{\mathrm{yp}}$ by the method proposed. For shaping $S_{\mathrm{yp}}$ a pair of complex zeros in $H_{\mathrm{R}}$

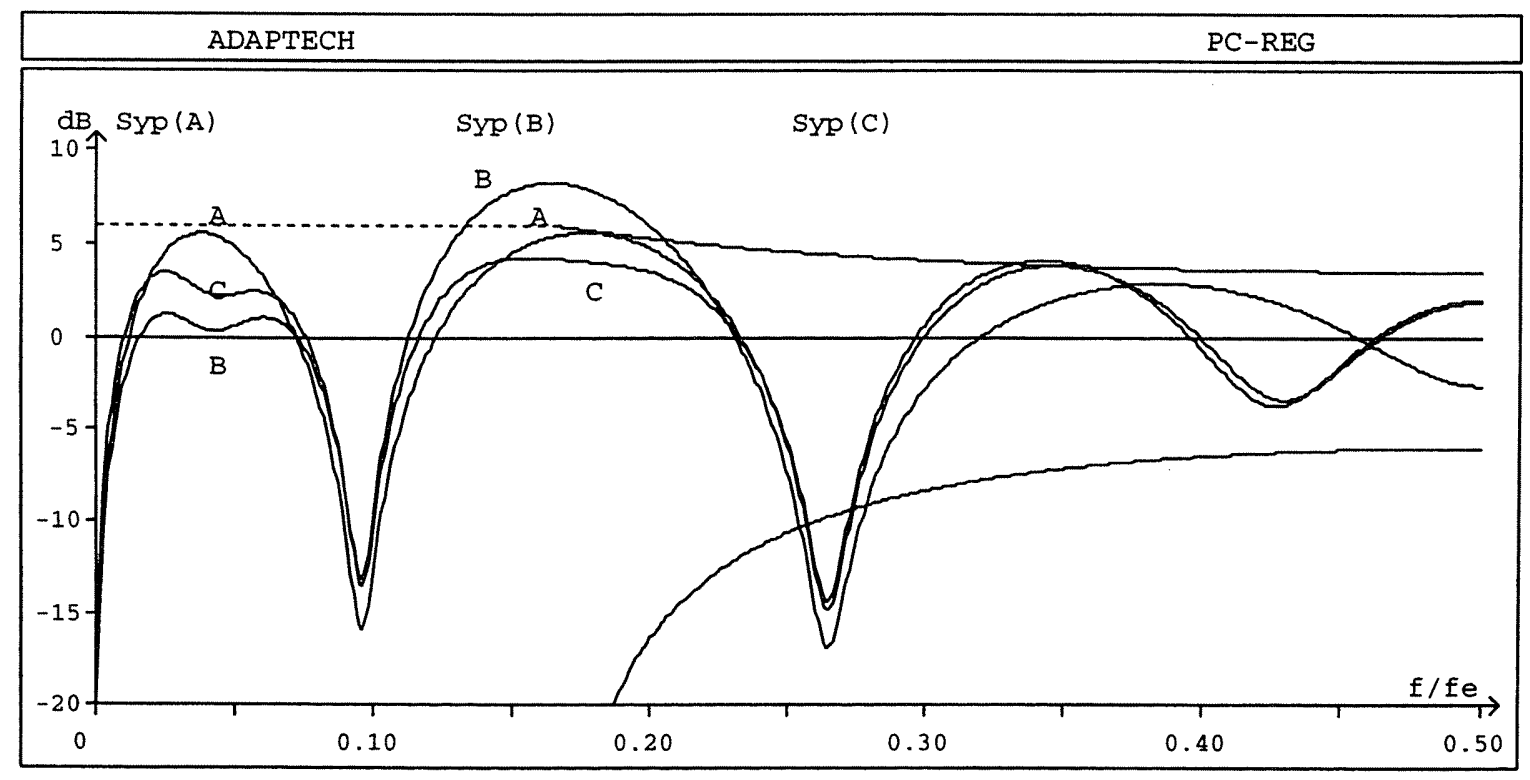

Fig. 3. Evolution of the output sensitivity function in different steps of design. 


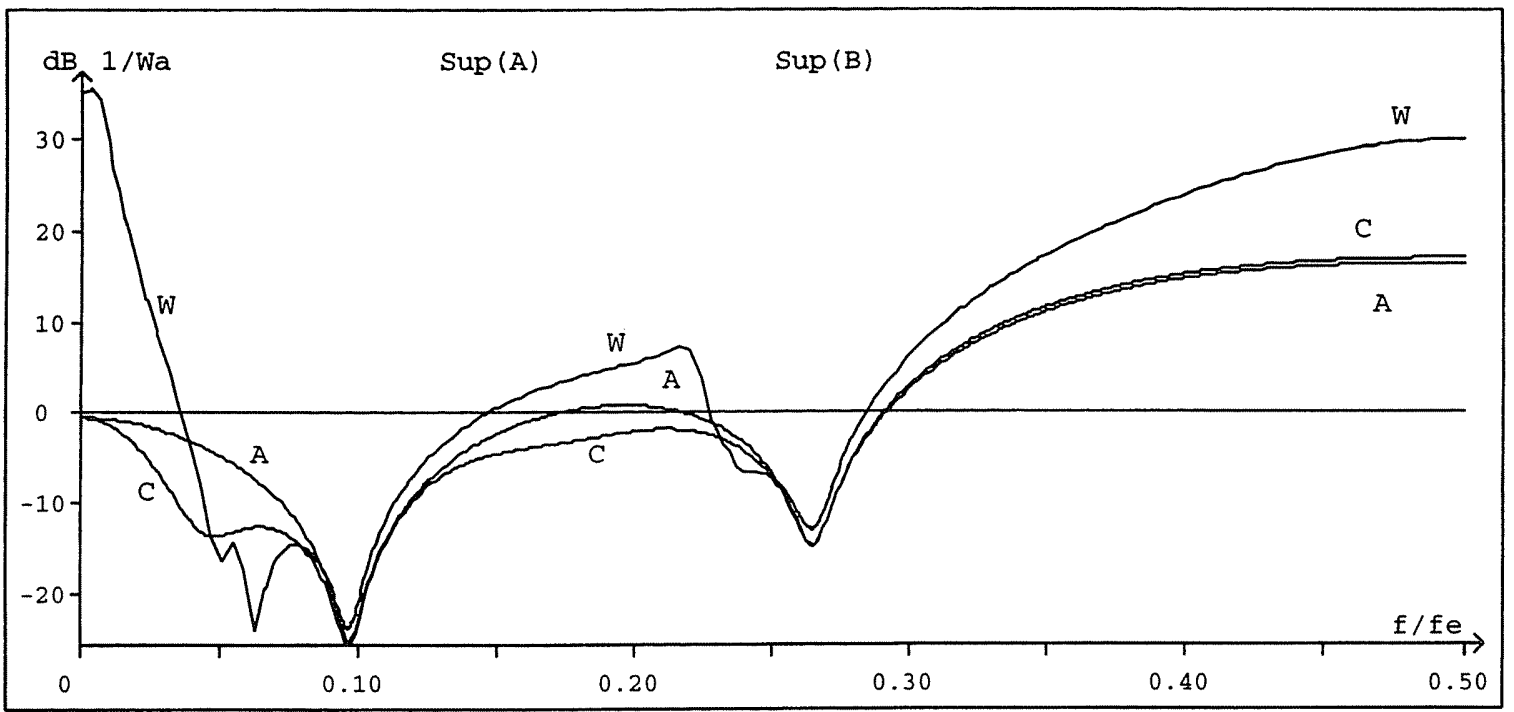

Fig. 4. Input sensitivity function $S_{u p}$ and inverse of additive uncertainty.

$\left(\omega_{0}=22.5 \mathrm{rad} / \mathrm{s} \backslash\right.$ and $\left.\zeta=0.25\right)$ and the auxiliary real poles $\left(1-0.2 q^{-1}\right)^{9}\left(1-0.55 q^{-1}\right)$ are used. The introduction of this leads to the sensitivity functions represented by the curve $\mathrm{C}$ in Figs 3 and 4.

The flexible system is now robustly stable in the presence of load variations (despite a small violation of the sufficient condition (9)) and it also meets the specifications for the nominal model (except for $S_{\mathrm{up}}$ in the high frequencies). However, for the loaded cases the rejection time of the disturbances are too long and the step responses are under damped. This means that robust performance is not yet achieved. Since the disturbance is filtered by $1 / A$, for output disturbance analysis it will be very interesting to look at the magnitude of $S_{\mathrm{yp}}$ multiplied by $1 / A$ for the loaded cases $(S /$ $P$ ). From Fig. 5 (curve $\mathrm{C}$ ) it can be concluded that the large value of $S / P$ (about $35 \mathrm{~dB}$ ) may be the cause of insufficient damping for the loaded cases. Again, by adding a pair of complex zeros in $H_{\mathrm{S}}$ we will be able to reduce this maximum by $10 \mathrm{~dB}$ (Fig. 5, curve D). In this way the rejection of disturbances for the loaded cases will be significantly improved. It should be men-

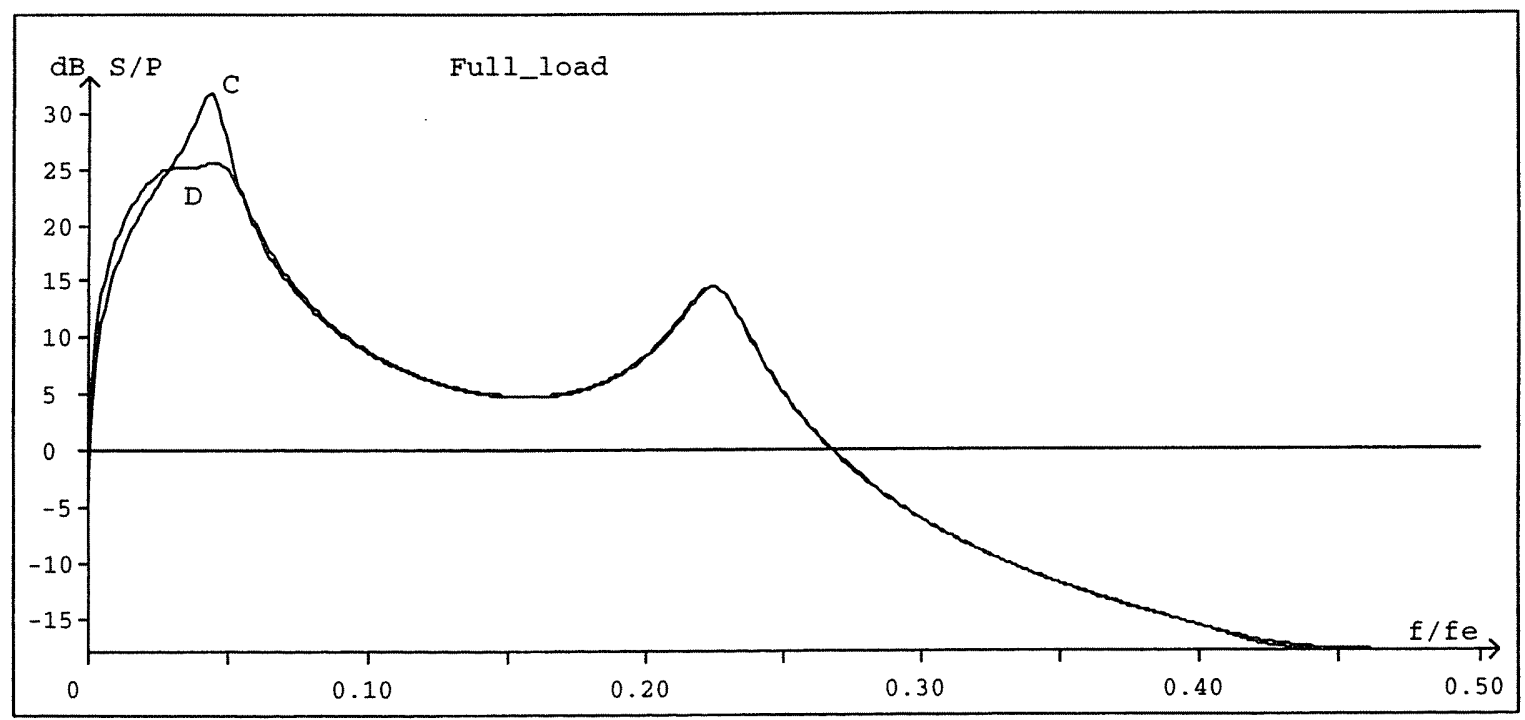

Fig. 5. $S_{\mathrm{yp}}$ filtered by $1 / A(S / P)$. 
tioned that after adding a pair of complex zeros in $H_{\mathrm{S}}$ the output sensitivity function shaping procedure presented in Section 5 is carried out in order to bring $S_{\mathrm{yp}}$ within the defined template.

From Fig. 4 (curve C) it is found that the magnitude of $S_{\text {up }}$ at high frequencies is too large and the constraint on $S_{\text {up }}$ is not satisfied. Therefore a term of the form $\left(1+z^{-1}\right)$ should be added in $H_{\mathrm{R}}$ to open the loop at high frequencies (Fig. 6). Table 1 shows different steps of controller design. In each step the zeros and poles which should be added to $H_{\mathrm{R}}, H_{\mathrm{S}}$ and $P_{\mathrm{F}}$ are shown. The complex zeros are presented in the form of the natural frequency and damping factor. After satisfying the conditions on $S_{\mathrm{yp}}$ and $S_{\mathrm{up}}$, the poles and zeros of the controller which are close to each other are cancelled. Then the prefilter is chosen as $T\left(q^{-1}\right)=R(1)$.

Table 1. The different steps of controller design.

\begin{tabular}{llll}
\hline & $H_{\mathrm{R}}$ & $H_{\mathrm{S}}$ & $P_{\mathrm{F}}$ \\
\hline $\mathrm{A}$ & - & $\left(1-z^{-1}\right)$ & - \\
$\mathrm{B}$ & - & $\omega_{0}=5.8 ; \zeta=.4$ & $\left(1-.2 z^{-1}\right)^{8}$ \\
$\mathrm{C}$ & $\omega_{0}=22.5$ & - & $\left(1-.2 z^{-1}\right)$ \\
& $\zeta=.25$ & & $\left(1-.55 z^{-1}\right)$ \\
$\mathrm{D}$ & - & $\omega_{0}=8.2 ; \zeta=.5$ & $\left(1-.55 z^{-1}\right)^{2}$ \\
$\mathrm{E}$ & $\left(1+z^{-1}\right)$ & - & - \\
\hline
\end{tabular}

The tracking reference model is a second order system with $\omega_{0}=12.5$ and $\zeta=0.75$. This natural frequency corresponds to the first vibration mode of the unloaded model. It is chosen in order to meet the rise time specification. This choice is not critical.

The final RST controller which meets almost all of the specifications is as follows:

$$
\begin{aligned}
& R\left(q^{-1}\right)=0.401602-1.079378 q^{-1}+0.284895 q^{-2} \\
& +1.358224 q^{-3}-0.986549 q^{-4}-0.271961 q^{-5} \\
& +0.306937 q^{-6} \\
& S\left(q^{-1}\right)=1-1.031142 q^{-1}-0.995182 q^{-2} \\
& +0.752086 q^{-3}+0.710744 q^{-4}-0.242297 q^{-5} \\
& -0.194209 q^{-6} \\
& T\left(q^{-1}\right)=0.013769
\end{aligned}
$$

The design of the controller as well as the simulations have been carried out using the PC-REG and PCREG/TR software [8].

\section{Simulation Results}

Figures 6-15 show the different characteristics of the controller and of the closed loop system in the presence of load variations. The performances of the controller are summarised in Table 2.

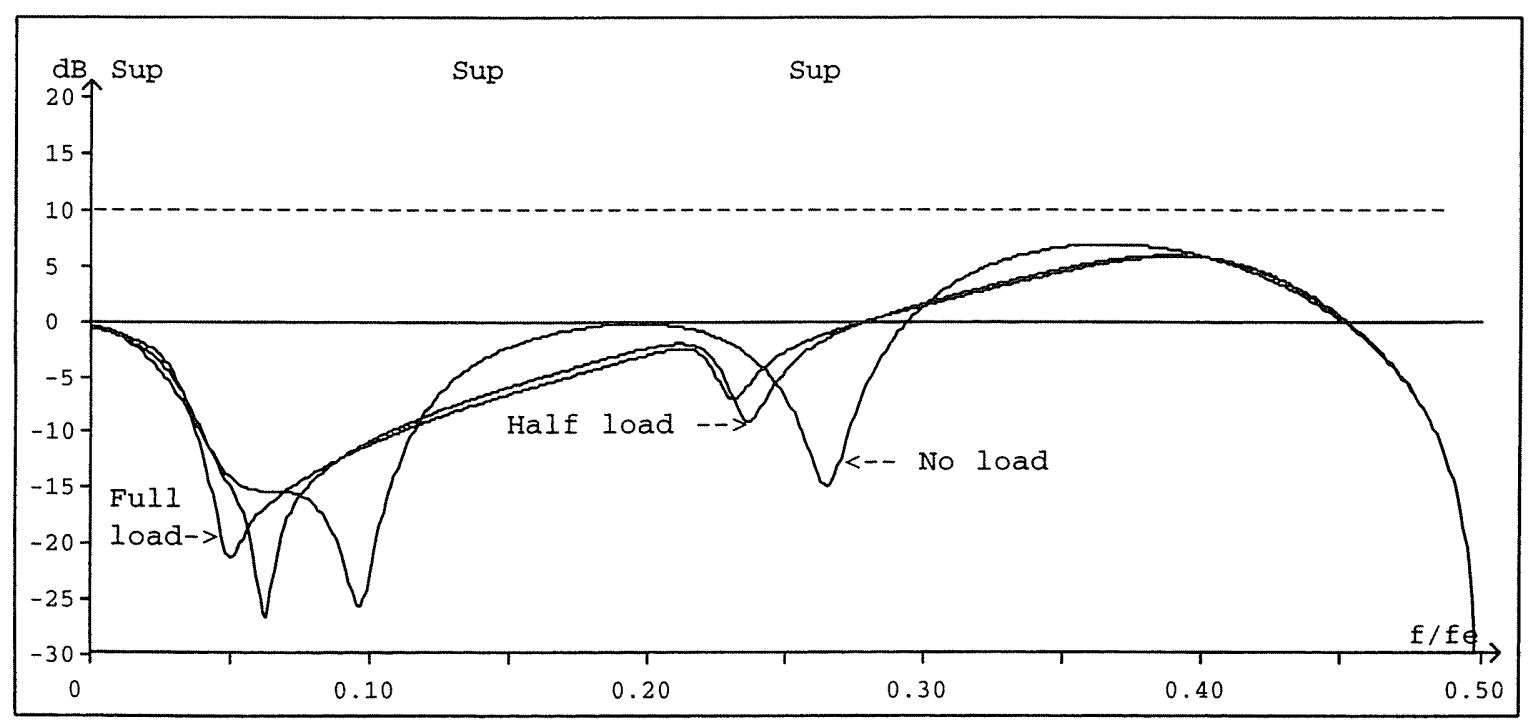

Fig. 6. Input sensitivity functions. 


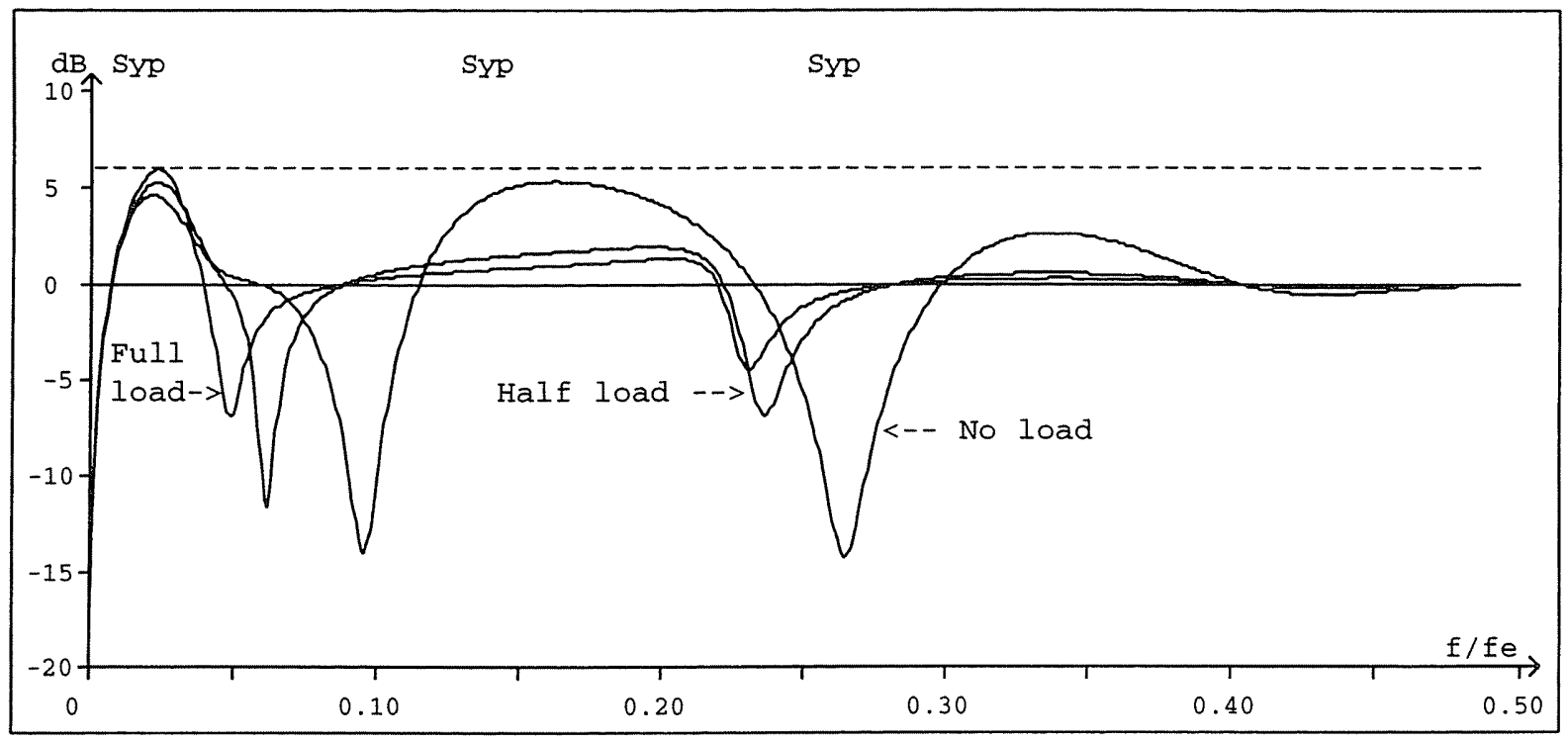

Fig. 7. Output sensitivity functions.

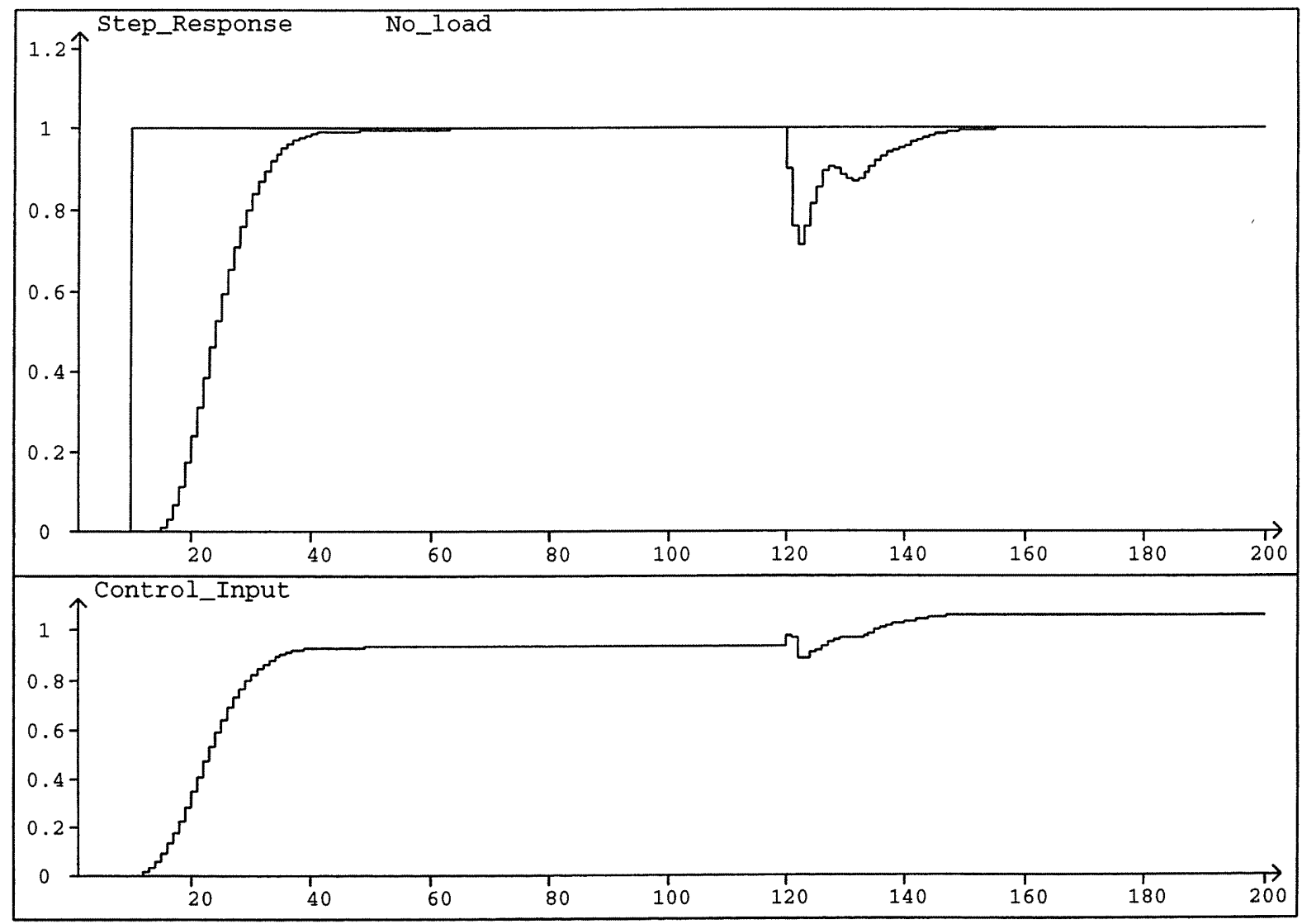

Fig. 8. Step and disturbance responses (no load case). 


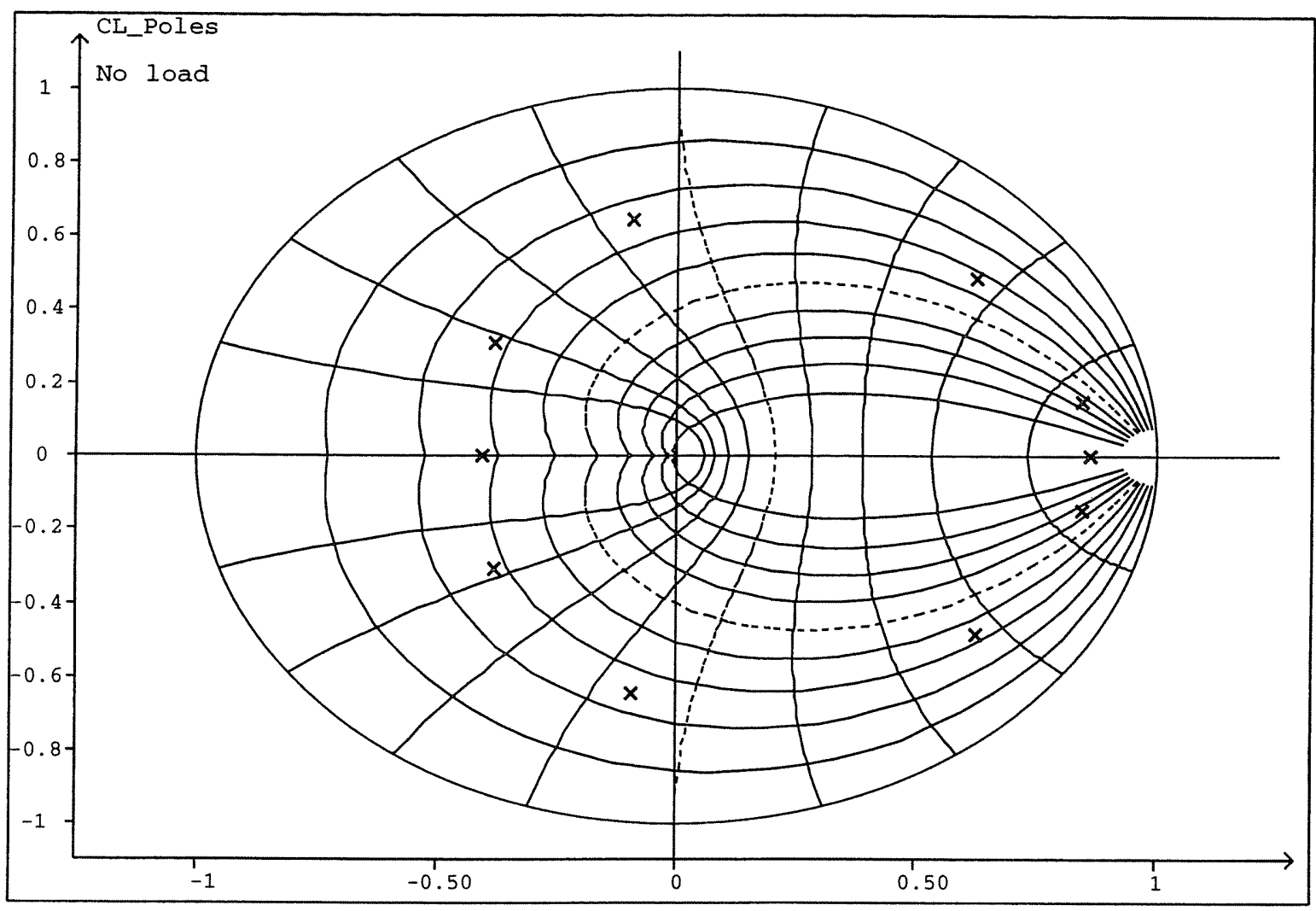

Fig. 9. Poles chart (no load case).

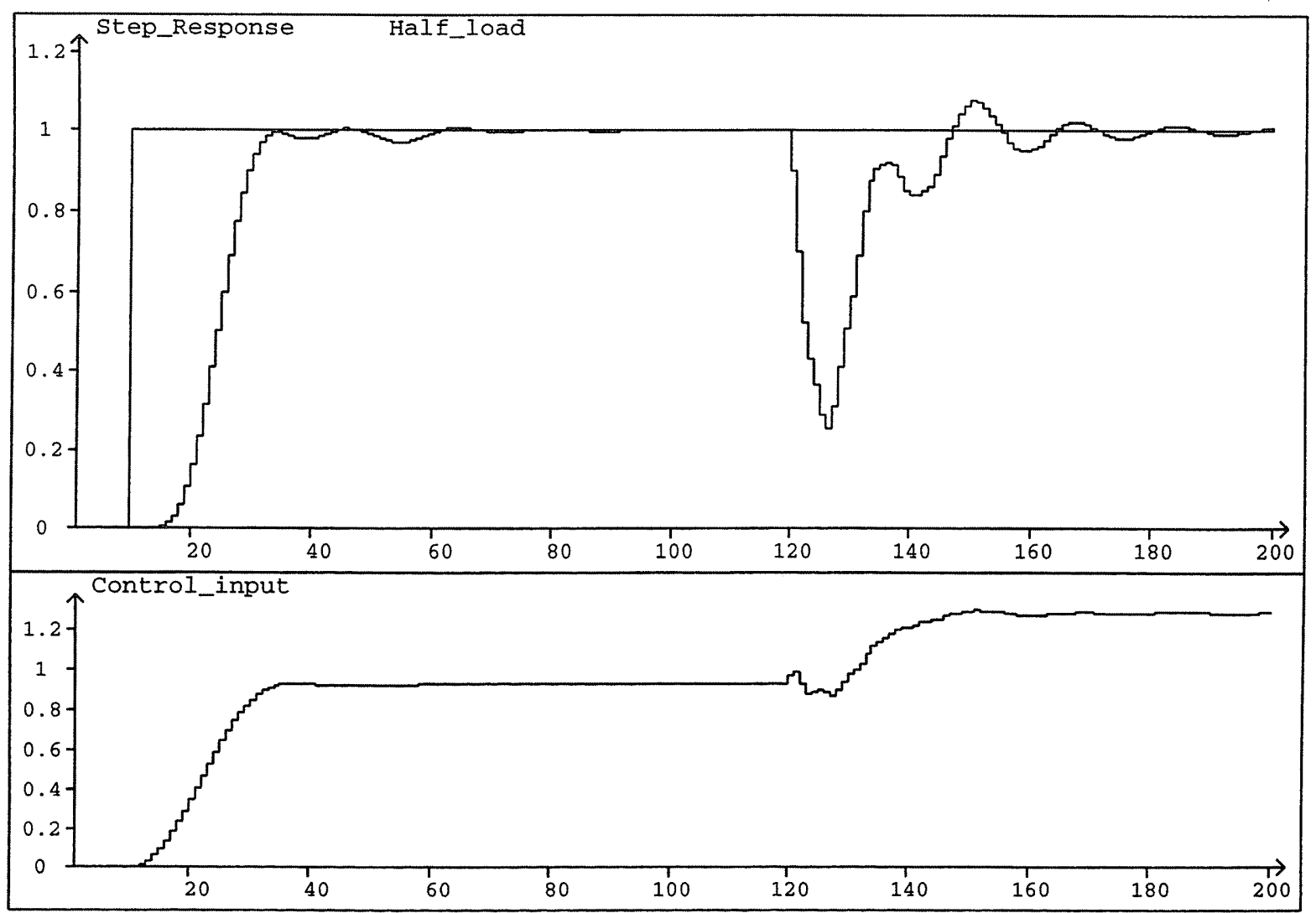

Fig. 10. Step and disturbance responses (half load case). 


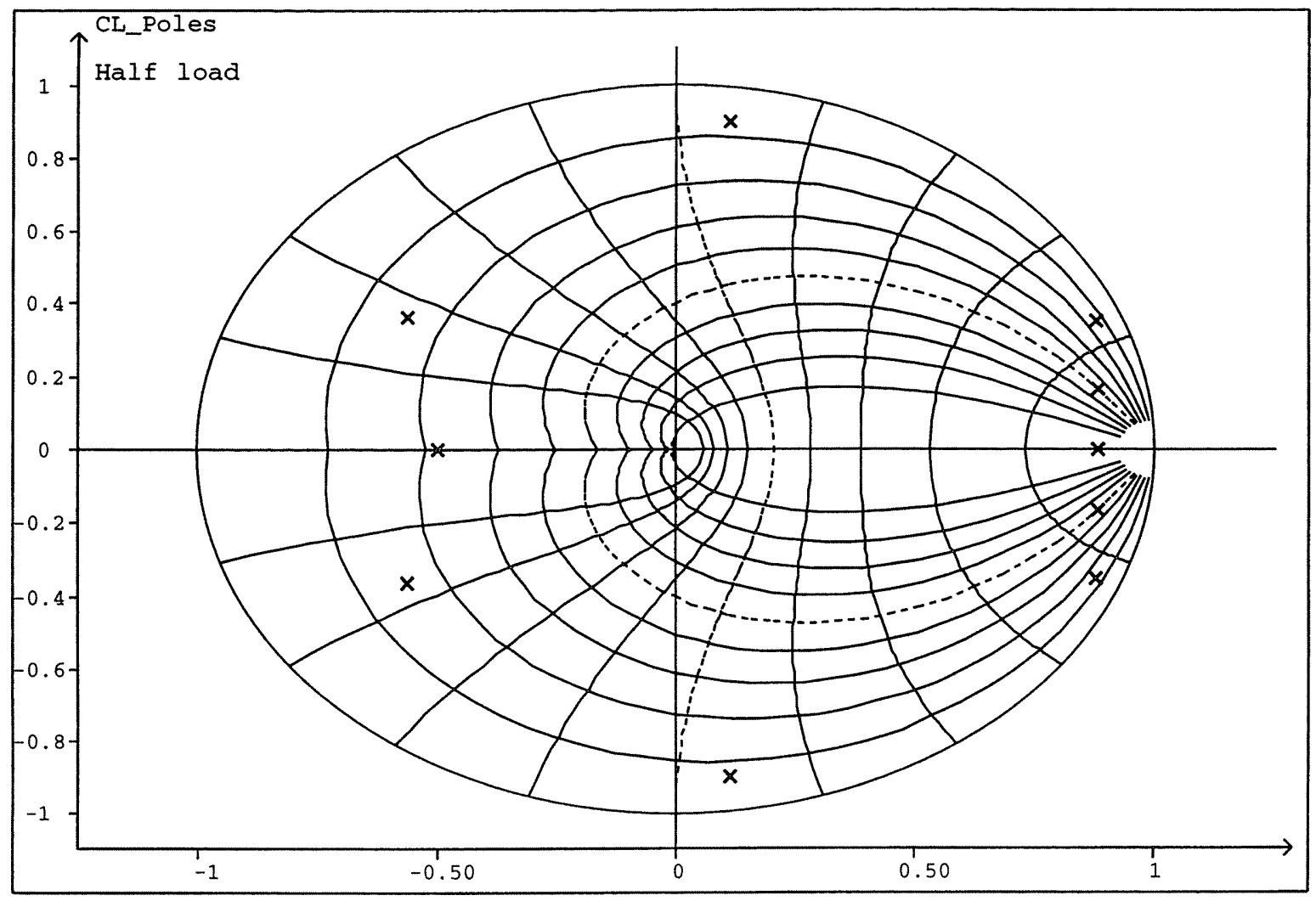

Fig. 11. Poles chart (half load case).



Fig. 12. Step and disturbance responses (full load case). 


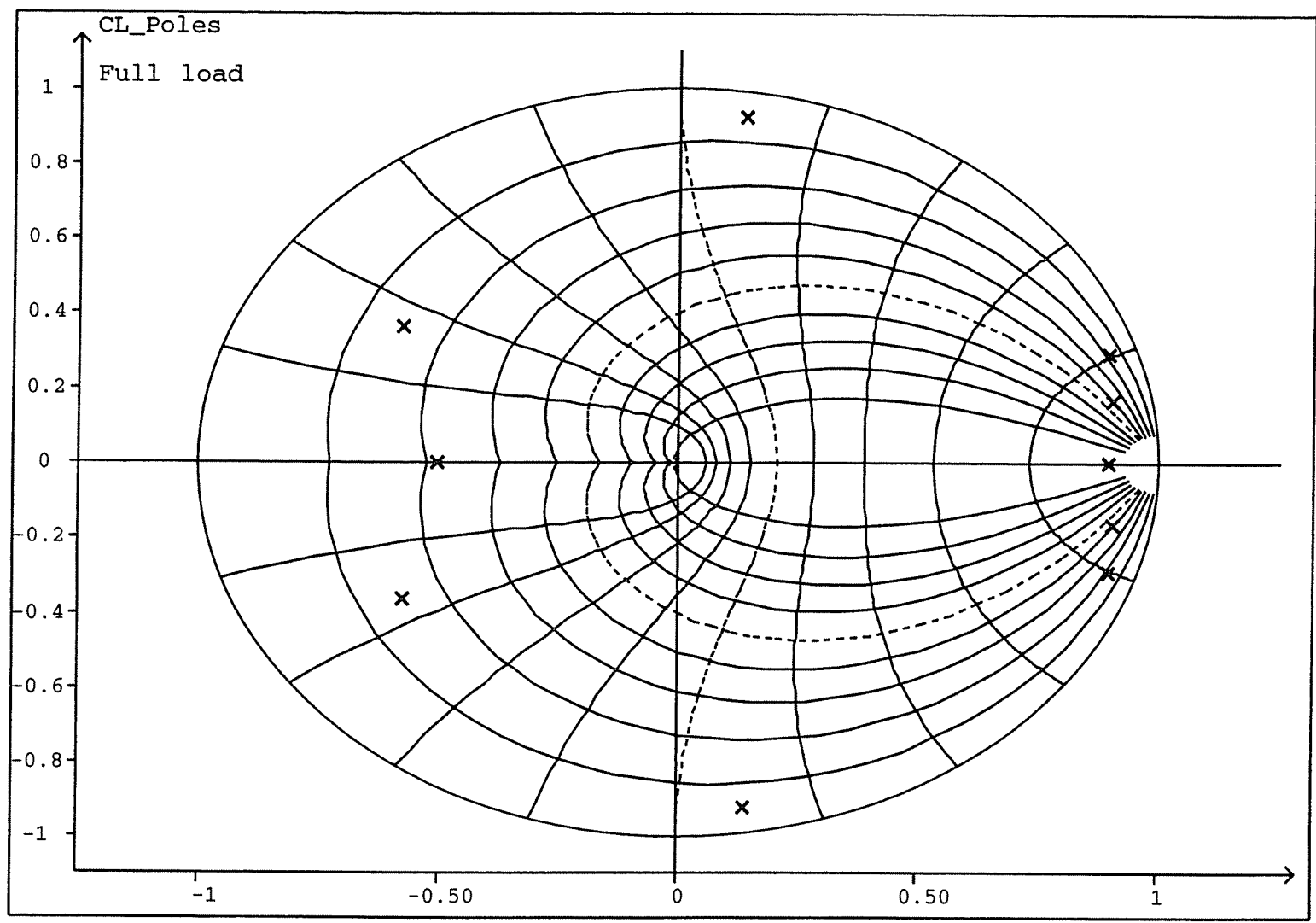

Fig. 13. Poles chart (full load case).

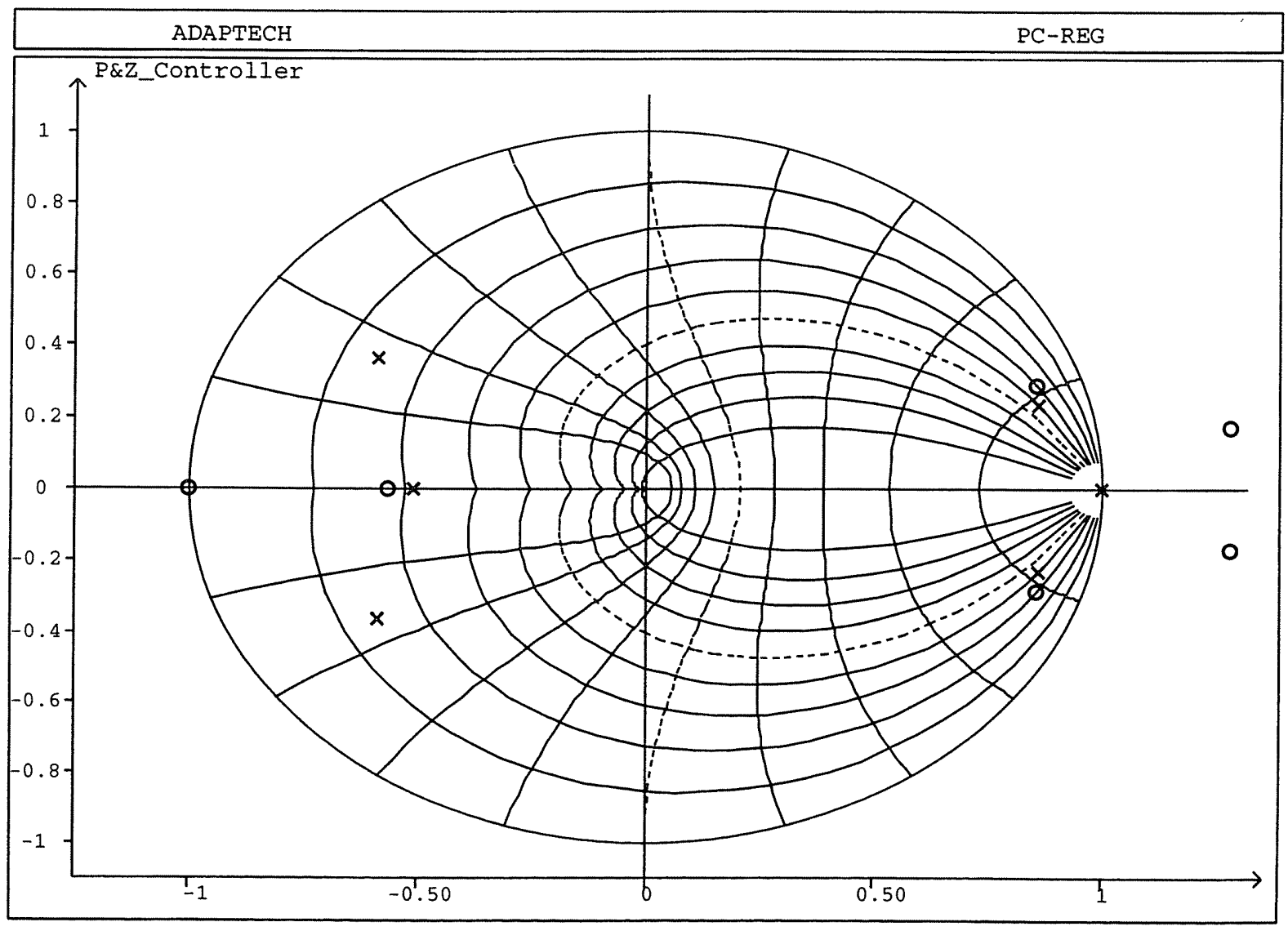

Fig. 14. Poles and zeros of controller. 


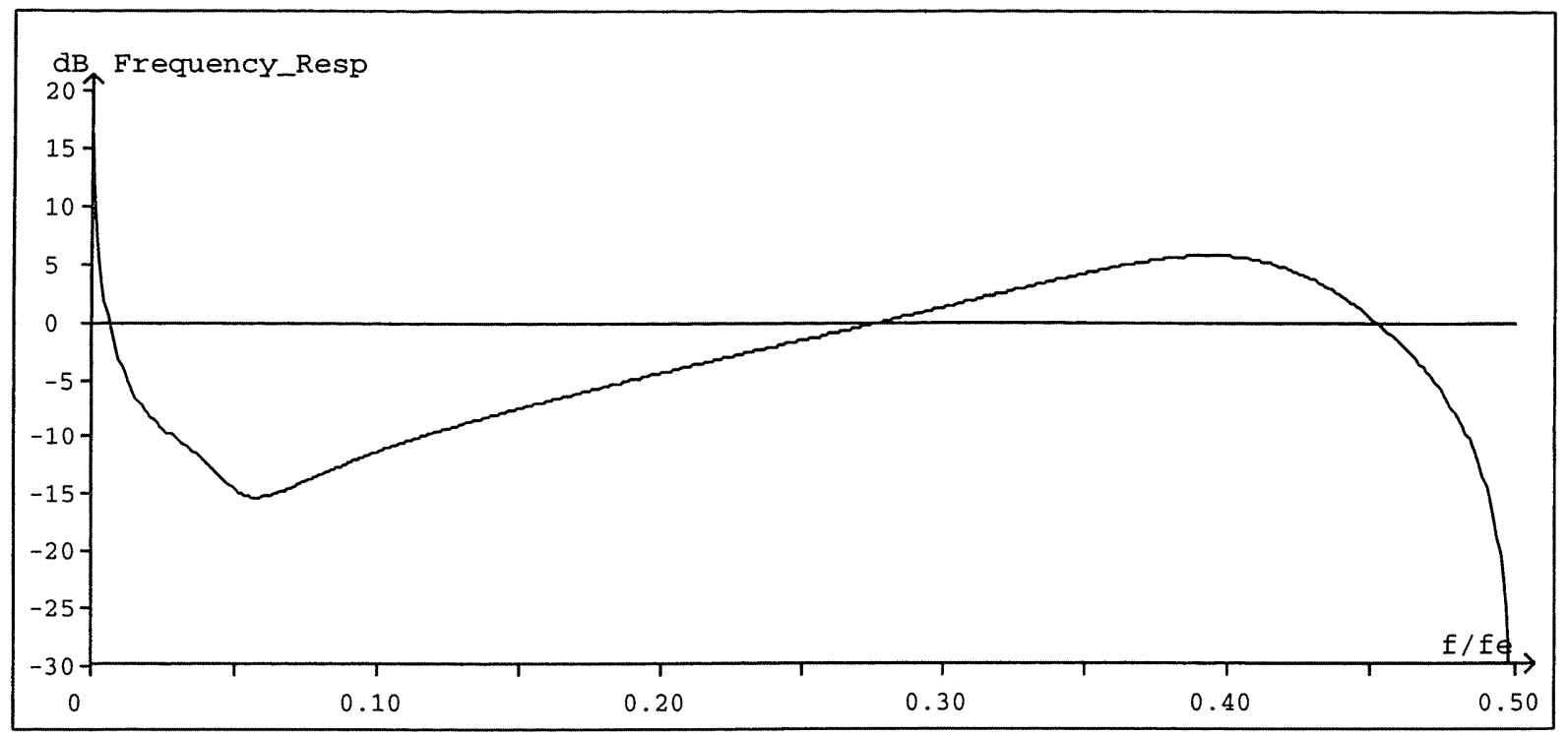

Fig. 15. Controller frequency response.

Table 2. The simulation results.

\begin{tabular}{lccc}
\hline & No load & Half load & Full load \\
\hline Rise time (s) & 1.06 & 0.90 & 0.93 \\
Over shoot (\%) & 0 & 2.03 & 10.02 \\
Rej. of dist. (s) & 1.11 & 1.24 & 0.89 \\
Delay margin (s) & 0.043 & 0.100 & 0.487 \\
Modulus margin (dB) & -5.33 & -5.32 & -5.98 \\
Attenuation band (Hz) & 0.164 & 0.170 & 0.164 \\
S $_{\text {up }}$ max (dB) & 5.90 & 5.80 & 5.79 \\
\hline
\end{tabular}

\section{Conclusions}

It has been shown in this paper that the control of flexible transmissions in the presence of large load variations can be obtained using a robust digital controller designed by pole placement combined with sensitivity function shaping. This method allows us to take into account iteratively, both robust stability conditions and robust performance specifications.

\section{References}

1. Landau ID, Rey D, Karimi A, Voda A, Franco A. A flexible transmission system as a benchmark for robust digital control. European J Control 1995; 1:77-96
2. Landau ID, Rolland F, Cyrot Ch, Voda A. Digital robust control. The combined pole placement/sensitivity shaping method. 1993 Report 94-009. Presented at the Summer Control School on Robustness Analysis and Design of Robust Controllers, Grenoble. French version published in 'La Robustesse', A. Oustaloup (Ed.), Hermes, Paris, 1995

3. Landau ID, Cyrot Ch, Rey D. Robust control design using the combined pole placement/sensitivity function shaping method. Proceedings ECC, 1993.

4. Landau ID. System Identification and Control Design. Prentice Hall, Englewood Cliffs, NJ, 1990

5. Doyle CJ, Francis BA, Tannenbaum AR. Feedback Control Theory, MacMillan, New York, 1992

6. Kwakernaak $\mathrm{H}$. Robust control and $\mathrm{H} \infty$ optimisation. Automatica, vol. 29, 2, 1993, 255-273

7. Landau ID. Robust digital control of systems with time delay. International Journal of Control, June 1995

8. Adaptech, PC-REG, PC-REG/TR Users' manuals, Version 5, 1993, 4, rue du Tour de l'Eau, St-Martin dHères, France 